\title{
Solid-Phase Synthesis of Alkanethiols for the Preparation of Self- Assembled Monolayers
}

\author{
Ratmir Derda ${ }^{\dagger}$, Daniel J. Wherritt $§$, and Laura L Kiessling ${ }^{\dagger} \neq$ \\ $\uparrow$ Departments of Chemistry University of Wisconsin, Madison, Wisconsin 53706 \\ $\$$ Departments of Biochemistry, University of Wisconsin, Madison, Wisconsin 53706
}

\begin{abstract}
Self-assembled monolayers (SAMs) of alkanethiols (ATs) on gold can be used to fabricate surfaces for nanoscience and biology. The chemical structure of the interface can be tailored simply by modifying the AT head group. To streamline access to different precursor ATs, we developed a general solid-phase synthetic route. A key feature of this route is the use of a modified resin containing an AT-linker ("AT-resin") because it minimizes purification steps. The precursor to AT-resin was prepared in 5 steps, and all of the synthetic intermediates are stable solids that can be purified by crystallization. Accordingly, the AT-resin can be prepared on a multi-gram scale. The utility of ATresin was evaluated by using it to generate a variety of ATs. For example, ATs presenting different types of integrin-binding ligands (linear and cyclic RGD derivatives) were prepared and used to form arrays of SAMs that support cell adhesion. Additionally, AT-resin also provides a starting point for the synthesis of ATs presenting reactive groups (e.g., an amine-reactive AT or a maleimidecontaining alkanedisulfide) or protein immobilization tags (e.g., biotin-AT). Thus, our synthetic strategy provides a convenient and flexible means for the synthesis of the necessary building blocks for custom SAMs and SAM arrays.
\end{abstract}

The spontaneous assembly of long-chain alkanethiols (ATs) on gold provides a powerful, yet simple, means of controlling surface properties at the molecular level. ${ }^{1,2}$ The molecular structure and physical properties of the resultant self-assembled monolayers (SAMs) are dictated by the chemical structure of the precursor ATs. Accordingly, the fabrication of monolayers with tailored properties hinges on access to chemically diverse ATs. ${ }^{3-6}$ We present a general synthetic approach to ATs to streamline access to structurally diverse SAMs.

SAMs can be fabricated by using two general approaches: assembly after conjugation (AAC) and conjugation after assembly (CAA) (Figure 1). In the CAA strategy, the desired moieties are attached to reactive SAMs through a series of heterogeneous reactions to form individual SAMs ${ }^{3,5,6}$ or arrays of SAMs. ${ }^{3}$ In the AAC strategy, a solution of AT presenting a desired terminal functionality is used to form SAMs on gold in a single step via immersion, ${ }^{1}$ spotting, 4 soft-lithography, 5,6 or scanning-probe lithography. ${ }^{7}$ If the ATs presenting the head-group of interest are not commercially available, they must be synthesized. Access to novel ATs often requires multiple synthetic and purification steps, and this need can serve a barrier that limits access to tailored SAMs. Indeed, a significant body of research using SAMs is confined to monolayers that can be formed by a handful of readily available ATs. The difficulty of

CORRESPONDING AUTHOR FOOTNOTE: Laura L. Kiessling, University of Wisconsin, Madison, WI 53706; E-mail: kiessling@chem.wisc.edu..

\$Present address: Department of Chemistry, The Ohio State University, Columbus, OH 43210

Supporting Information Available: Synthetic methods and experimental details for the development of methodology. This material is available free of charge via the Internet at http://pubs.acs.org. 
generating diverse ATs using current methods is also a challenge to the production of multicomponent SAM arrays, especially if each AT must be synthesized individually. Solidphase synthesis has facilitated the rapid assembly of related compounds, because multiple transformations can be conducted without laborious purification steps. ${ }^{8}$ We envisioned that both CAA and AAC strategies would benefit from a divergent solid-phase route to ATs (Figure $1)$.

A convergent solid-phase strategy for the synthesis of individual peptide-ATs 9,10 or small collections of peptide-ATs ${ }^{4}, 11$ has been developed. The key step involves the capping of an amine group on resinimmobilized peptide with a trityl-protected AT that bears a carboxylic acid. Though it has many useful attributes, this route is not divergent. A divergent approach is valuable because it can be used to rapidly generate ATs with different structures. In our approach, an immobilized amine-terminated AT serves as a starting point. This AT-resin can be used in multistep solid-phase synthesis to afford diverse ATs or symmetrical alkane disulfides (ADS). Byproducts are removed by simple resin washing, thereby expediting AT synthesis. The AT can be liberated from the resin using mild conditions to yield the target AT (s).

To generate the modified resin, we employed an AT possessing a protected primary amine (Scheme 1). This amine is masked with the tetrachlorophthalimide (TCP) protecting group, a group known to confer crystallinity. ${ }^{12}$ Indeed, all of the AT precursors (compounds $\mathbf{2 ,} 3$ and 4, Scheme 1) are stable solids that can be purified by crystallization. Thus the synthesis of ATresin is readily scalable to afford multi-gram quantities. In contrast, most reported oligo (ethylene glycol)-ATs and their precursors are viscous liquids,, 16 which require chromatography for their purification. Importantly, we found the crystallinity of TCP-bearing oligo(ethylene glycol) compounds to be quite general (see Table 1S, Supporting Information). Though we focused on synthesizing the tetra(ethylene glycol)-containing derivatives, we anticipate that this approach can be implemented to generate AT-resins with varying numbers of the oligo(ethylene glycol) moieties (i.e. $n=0-6$ ) as well as ATs possessing alkane chains of different lengths.

Using the synthetic route outlined, resin 1 was produced in an overall yield of $40 \%$. The product of the tetra(ethylene glycol) alkylation with commercially available 11-bromo-1-undecene (Scheme 1) was isolated via extraction and used directly in Mitsunobu reaction to yield alkene 2. The product was purified by consecutive crystallizations from solvents of opposing polarities (n-heptane and methanol). Radical addition of thioacetic acid afforded thioester 3; the acetyl group was removed by acidic hydrolysis to produce $\mathbf{4}$, which was crystallized from methanol. Thiol 4 was immobilized onto monomethoxytrityl-chloride (MMT-Cl) resin in quantitative yield; therefore, the loading level of resin 1 can be set to any value in the $0.1-0.8 \mathrm{mmol} / \mathrm{g}$ range (see Figure 1S, Supporting Information). This flexibility is beneficial for certain solid phase syntheses (e.g. the yields of multi-step peptide synthesis can improve with lower resin loading). 13 After immobilization of 4 , the TCP group was removed with hydrazine ${ }^{12}$ to afford resin 1.

Resin 1 provides a starting point for the solid-phase synthesis of different ATs. Our interest in using SAMs to explore cell adhesion and signaling led us to select as our initial target an AT that displays a moiety known to interact with a cell surface receptor. Specifically, we synthesized an AT displaying the integrin binding sequence Gly-Arg-Gly-Asp-Ser-Pro (GRGDSP), which can mediate cell adhesion. ${ }^{14}, 15$ SAMs displaying ATs that present this sequence have been well-characterized, ${ }^{16}$ thereby providing a benchmark with which to compare the activity of SAMs composed of our synthetic AT. To assemble the target AT, we employed resin 1 in automated peptide synthesis. ${ }^{17}$. Upon cleavage from the resin, the expected peptide-substituted AT was obtained. With this approach the AT is appended at the 
peptide C-terminus (Scheme 2a). Mrksich and co-workers have reported previously a solid phase route to an RGD-containing sequence with AT-moiety attached to peptide N-terminus. 9 Therefore, the two synthetic strategies complement each other and provide access to SAMs presenting peptide sequences in opposite orientations. Often it is not known a priori what orientation enables optimal epitope binding; thus, these general synthetic routes can be used in concert to dissect the role of the surface presentation on epitope recognition. ${ }^{18}$ Although binding to the RGD-motif does not require a free $\mathrm{N}$-terminus, some receptorpeptide interactions do. ${ }^{19}$ Other functions (e.g. proteolysis) also can be greatly influenced by the presence of an $\mathrm{N}$-terminal amino group. The complementarity of the two aforementioned solidphase strategies makes it possible to rapidly determine the AT attachment point that yields the best performance.

Our synthetic methodology is not limited to synthesis of linear peptide-substituted ATs; it can be applied to generate other types of AT conjugates. For example, any compound bearing a primary amine can be tethered to resin $\mathbf{1}$, through a squaric acid moiety. ${ }^{20}$ Because squarate coupling is mild and chemoselective, it can tolerate a variety of reactive groups, which makes it an excellent method for linking compounds rich in functional groups (e.g. carbohydratecontaining molecules). ${ }^{25-27}$ We used this approach to synthesize an AT containing a cyclic peptide known to interact with the integrin $\alpha_{v} \beta_{3} .{ }^{21}$ Following the reaction of dimethoxy squarate with 1 , commercially available $\mathrm{c}(\mathrm{RGDyK})$ was added in the presence of base (Scheme 1b). This coupling proceeded with high chemoselectivity; a free carboxylic acid, phenol and guanidinium moieties were all tolerated.

To evaluate the function of ATs generated by our solid-phase strategy, we fabricated SAMs from our synthetic ATs that present the Arg-Gly-Asp (RGD) sequence, 5, or cyclic RGD 6 for evaluating cell adhesion. We fabricated multicomponent arrays of SAMs starting from perfluoro-SAMs. ${ }^{4,}{ }^{11} \mathrm{UV}$ irradiation through a photomask defines the shape and location of the array elements. Solutions containing compounds $\mathbf{5}$ or $\mathbf{6}$ mixed with a non-adhesive AT, glucamine-AT, ${ }^{22}$ were spotted onto exposed gold array elements surrounded by a hydrophobic perfuoro-SAM. This two-step procedure generates an array of SAMs with individual elements that vary in the surface density of linear or cyclic-RGD peptide presented. To aid comparison (and alignment) of multiple arrays, each array was equipped with patterned gold features. When a solution of $\mathrm{KCN}$ is spotted onto peripheral array elements, the SAM-free surface is selectively etched to expose the glass (Figure 2, row marked "0"). ${ }^{23}$ The resulting regions were clearly apparent using an optical microscope, and provided reference points for alignment of the arrays tested.

To evaluate cell adhesion to the arrays, we employed the human melanoma cell line WM-115. These cells display the integrin $\alpha_{\mathrm{v}} \beta_{3}$ and therefore should bind both linear and cyclic RGDderived peptides. ${ }^{24,25}$ We found that WM-115 cells adhere to SAMs derived either from the linear or the cyclic RGD-presenting ATs. In contrast, the cells avoid surfaces lacking cellbinding epitopes (glass or perfluoro-SAM). The observed cell binding hinges on the specific epitope displayed on the AT. Specifically, cells do not adhere to the ligand-displaying surfaces in the presence of soluble RGDS peptide (Figure 2).These results indicate that our synthetic strategy yields ATs that form SAMs and support specific cell adhesion.

We postulated that our solid-phase route to ATs also would provide convenient access to precursor-ATs for use in the CAA strategy (Figure 1). A difficulty in generating precursor ATs is their intrinsic reactivity. An advantage of the monomethoxythrityl (MMT) AT-resin linkage is that it can be cleaved under mild acidic or oxidative conditions; it also is sensitive to heavy metal ions with affinity for thioether groups (e.g. $\left.\mathrm{Ag}^{+}, \mathrm{Hg}^{+}\right) .{ }^{26}$ We anticipated that the ability to employ appropriate and mild cleavage conditions would facilitate the preparation of ATs containing sensitive chemical functionalities. For example, AT-presenting amine-reactive 
squaric acid group, 7, can be liberated under mild conditions (2\% TFA, $2 \%$ ethanethiol in $\mathrm{CH}_{2} \mathrm{Cl}_{2}$ ) that minimize possible side reactions (Scheme 2c). Squarate-modified AT can be used to form SAMs with selective reactivity toward primary amines,. Biotin-presenting AT $\mathbf{8}$ is not sensitive to strong acids; therefore, it can be assembled on resin and released under conditions used to liberate peptide-ATs (Scheme 2d). Biotin-AT can be used to form SAMs for sitespecific protein immobilization. ${ }^{27}$ Thus, cleavage conditions can be designed to afford ATs that can be used for diverse purposes.

Some useful precursor ATs bear reactive moieties that are incompatible with the corresponding alkanethiols. SAMs of ATs bearing such groups can be prepared in one step from the corresponding alkanedisulfides (ADS), in which the thiol group is masked as a symmetrical disulfide. ${ }^{28}$ Resin 1 allows facile preparation of the symmetrical disulfides via oxidative cleavage conditions. For example, SAMs presenting a thiol-reactive maleimide group cannot be prepared from self-reactive maleimide-AT, but a maleimide-alkanedisulfide can be used as a monolayer precursor. In turn, it can be synthesized using resin $\mathbf{1}$ in just two steps (Scheme 2e). Coupling of a maleimide-containing carboxylic acid followed by acid-induced cleavage in the presence of mild oxidant $\left(\mathrm{I}_{2}\right)$ affords maleimide-containing alkanedisulfide. These cleavage conditions prevent conjugate addition of thiol to the maleimide (see Supporting Information). Furthermore, we stored the maleimide-ADS for extended periods ( $>1$ year) at room temperature (neat) with no significant changes in its purity (see Supporting Information).

In summary, we have demonstrated an efficient solid-phase strategy for the multi-step synthesis of ATs. The key reagent, the AT-resin 1, provides the means to go beyond the limited commercially available repertoire to a collection of tailored ATs. As with any solid-phase route, this strategy is amenable to combinatorial synthesis: The AT-resin could be used as a starting point for the rapid synthesis of the libraries of ATs via parallel or split and pool methodologies. 29 Although our examples focus on the synthesis of the SAM-precursors for biological applications, we anticipate that our route to ATs will benefit other applications that rely on defined surfaces (e.g. molecular electronics) or multicomponent surfaces (e.g., arrays of bilayers ${ }^{30}$ ). Our strategy can also be used to access other types of SAM precursors (e.g., functionalized thiol-biphenyls, ${ }^{31}, 32$ alkane-silanes, ${ }^{33,} 34$, alkenes and other cycloaddition precursors, ${ }^{41,42}$ etc). The strategy demonstrated here offers a general solution: A monolayer precursor is embedded in the solid-phase linker, while the surface-reactive functionality is masked within the resin-anchoring functionality. We anticipate the general approach outlined here will enable the synthesis of monolayer precursors and therefore the fabrication of new types of SAMs.

\section{Supplementary Material}

Refer to Web version on PubMed Central for supplementary material.

\section{Acknowledgment}

This research was supported by DARPA (N66001-03-1-8932), NIH (AI054092) and the University of Wisconsin, Materials Research Science and Engineering Center (DMR-0520527). We thank the W. M. Keck Foundation for supporting the Center for Chemical Genomics. We thank P. J. Belshaw for use of equipment. We acknowledge A. Tam for the sample of maleimide-butyric acid. We thank B. P. Orner for helpful discussions.

\section{References}

1. Bain CD, Troughton EB, Tao YT, Evall J, Whitesides GM, Nuzzo RG. J. Am. Chem. Soc 1989;111 (1):321-335.

2. Love JC, Estroff LA, Kriebel JK, Nuzzo RG, Whitesides GM. Chem. Rev 2005;105(4):1103-1169. [PubMed: 15826011] 
3. Brockman JM, Frutos AG, Corn RM. J. Am. Chem. Soc 1999;121(35):8044-8051.

4. Orner BP, Derda R, Lewis RL, Thomson JA, Kiessling LL. J. Am. Chem. Soc 2004;126(35):1080810809. [PubMed: 15339142]

5. Kane RS, Takayama S, Ostuni E, Ingber DE, Whitesides GM. Biomaterials 1999;20(2324):2363-2376. [PubMed: 10614942]

6. Wilbur JL, Kumar A, Kim E, Whitesides GM. Adv. Mater 1994;6(78):600-604.

7. Piner RD, Zhu J, Xu F, Hong SH, Mirkin CA. Science 1999;283(5402):661-663. [PubMed: 9924019]

8. Merrifield RB. J. Am. Chem. Soc 1963;85(14):2149-54.

9. Houseman BT, Mrksich M. J. Org. Chem 1998;63(21):7552-7555. [PubMed: 11672416]

10. Miller JS, Bethencourt MI, Hahn M, Lee TR, West JL. Biotechnol. Bioeng 2006;93(6):1060-1068. [PubMed: 16444742]

11. Derda R, Li L, Orner BP, Lewis RL, Thomson JA, Kiessling LL. ACS Chem. Biol. 2007, in press, DOI: $10.1021 / \mathrm{cb} 700032 \mathrm{u}$.

12. Debenham J, Rodebaugh R, FraserReid B. Liebigs Ann./Recueil 1997;(5):791-802.

13. Kent SBH. Annu. Rev. Biochem 1988;57:957-989. [PubMed: 3052294]

14. Pierschbacher MD, Ruoslahti E. Nature 1984;309(5963):30-33. [PubMed: 6325925]

15. Ruoslahti E, Pierschbacher MD. Science 1987;238(4826):491-497. [PubMed: 2821619]

16. Roberts C, Chen CS, Mrksich M, Martichonok V, Ingber DE, Whitesides GM. J. Am. Chem. Soc 1998;120(26):6548-6555.

17. Merrifield RB. Science 1965;150(693):178-85. [PubMed: 5319951]

18. Luk YY, Tingey ML, Dickson KA, Raines RT, Abbott NL. J. Am. Chem. Soc 2004;126(29):90249032. [PubMed: 15264835]

19. Behanna HA, Donners J, Gordon AC, Stupp SI. J. Am. Chem. Soc 2005;127(4):1193-1200. [PubMed: 15669858]

20. Tietze LF, Arlt M, Beller M, Glusenkamp KH, Jahde E, Rajewsky MF. Chem. Ber 1991;124(5):12151221.

21. Haubner R, Gratias R, Diefenbach B, Goodman SL, Jonczyk A, Kessler H. J. Am. Chem. Soc 1996;118(32):7461-7472.

22. Luk YY, Kato M, Mrksich M. Langmuir 2000;16(24):9604-9608.

23. Kumar A, Biebuyck HA, Abbott NL, Whitesides GM. J. Am. Chem. Soc 1992;114(23):9188-9189.

24. Carlson CB, Mowery P, Owen RM, Dykhuizen EC, Kiessling LL. ACS Chem. Biol 2007;2(2):119127. [PubMed: 17291050]

25. Allman R, Cowburn P, Mason M. Eur. J. Cancer 2000;36(3):410-422. [PubMed: 10708944]

26. Green, TW.; Wuts, PGM. Protective Groups in Organic Synthesis. 4th. Wiley; 2006.

27. Spinke J, Liley M, Schmitt FJ, Guder HJ, Angermaier L, Knoll W. J. Chem. Phys 1993;99(9):70127019.

28. Biebuyck HA, Bain CD, Whitesides GM. Langmuir 1994;10(6):1825-1831.

29. Lam KS, Salmon SE, Hersh EM, Hruby VJ, Kazmierski WM, Knapp RJ. Nature 1991;354(6348): 82-84. [PubMed: 1944576]

30. Castellana ET, Cremer PS. Surf. Sci. Rep 2006;61(10):429-444.

31. Sabatani E, Cohenboulakia J, Bruening M, Rubinstein I. Langmuir 1993;9(11):2974-2981.

32. Ulman A. Acc. Chem. Res 2001;34(11):855-863. [PubMed: 11714257]

33. Netzer L, Iscovici R, Sagiv J. Thin Solid Films 1983;99(13):235-241.

34. Wasserman SR, Tao YT, Whitesides GM. Langmuir 1989;5(4):1074-1087. 


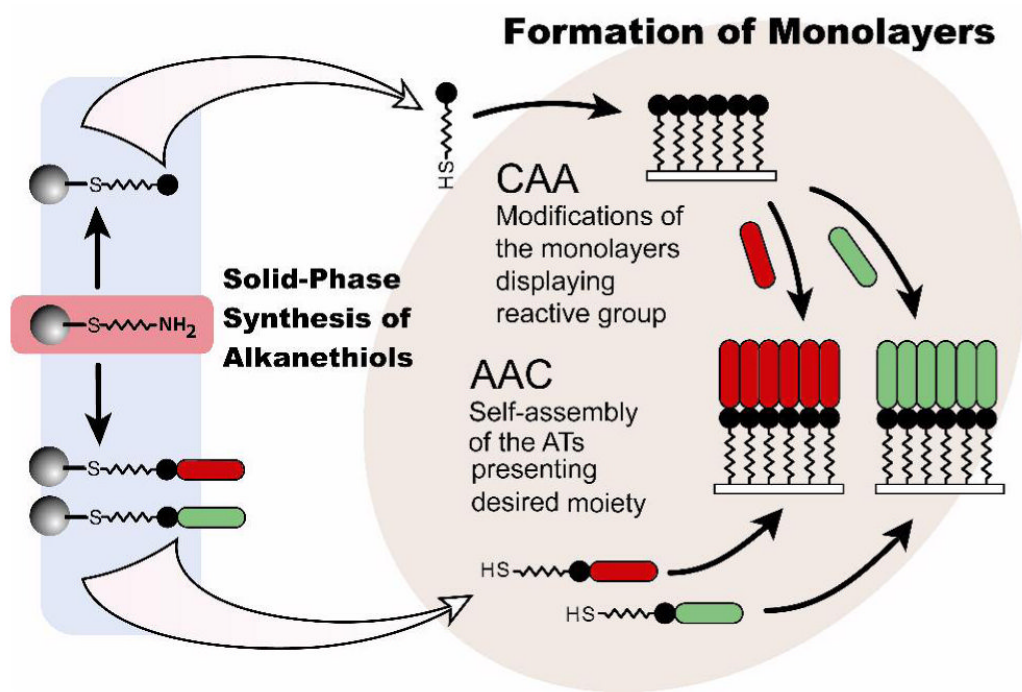

Figure 1.

Current approaches to fabrication of SAMs can be classified as "conjugation after assembly" (CAA) and "assembly after conjugation" (AAC). Solid-phase methodology can be used to synthesize an AT to use in AAC strategy, or, it can be used to synthesize ATs with diverse reactive head groups (denoted with black circle) for CAA strategy. 
<smiles>C=CC(C)(C)CBr</smiles>

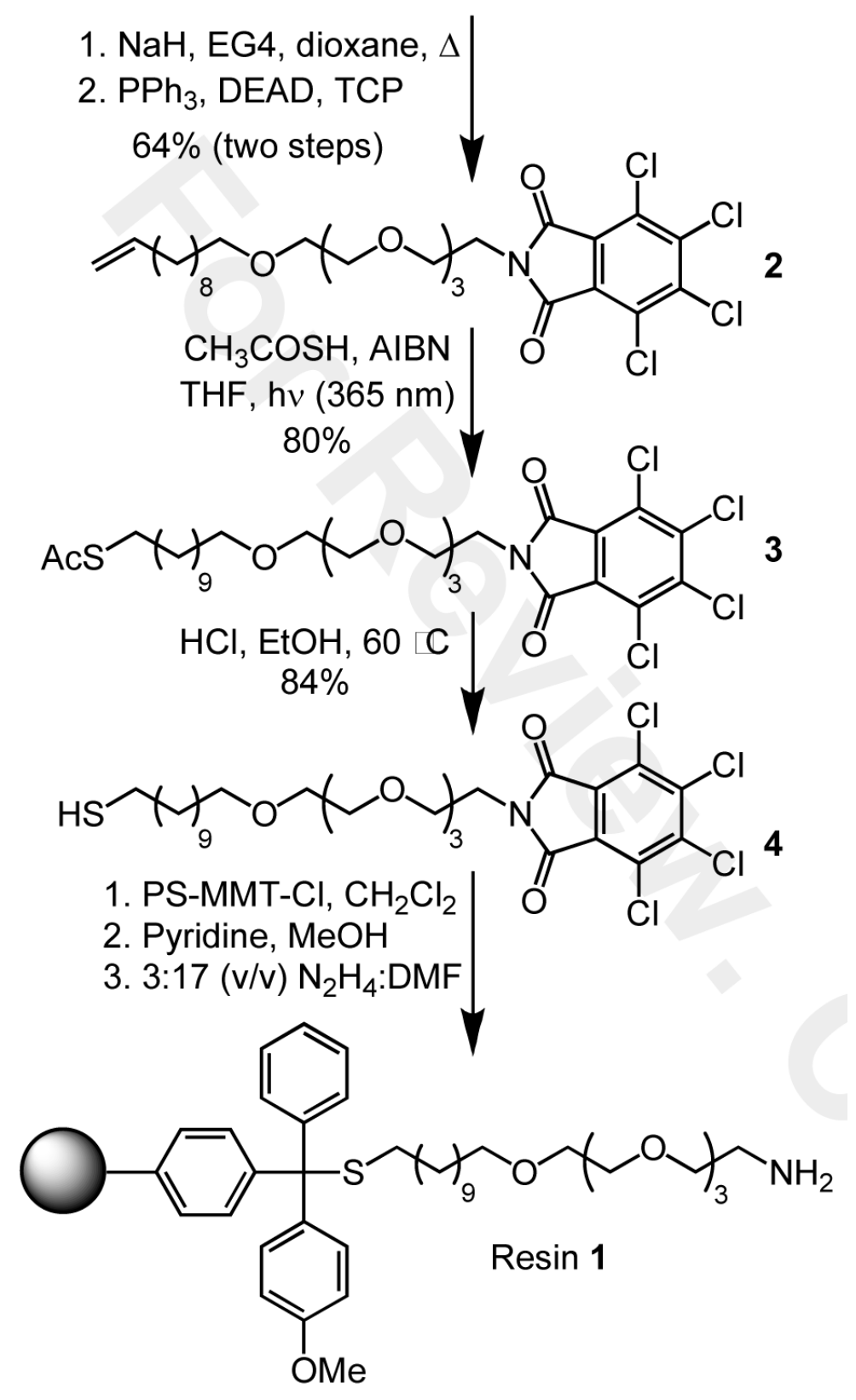

Scheme 1.

Synthesis of the AT-resin. Abbreviations used: EG4—tetra(ethylene glycol), DEAD—diethyl azodicarboxylate; TCP—tetrachlorophthalimide; AIBN—azobisisobutyronitrile; THFtetrahydrofuran; EtOH — ethanol; Ac—acetyl; PS-MMT-Cl—polystyrene-bound monomethoxytrityl chloride; $\mathrm{MeOH}$ - methanol; $\mathrm{DMF}-N, N$-dimethylformamide 
1. Automated peptide

a. $\operatorname{Resin} 1$ synthesis

2. $92.5 \%$ TFA, $2.5 \%$ TIS

$2.5 \%$ EDT, $2.5 \% \mathrm{H}_{2} \mathrm{O}$

b. Resin 1<smiles></smiles>

c. Resin 1

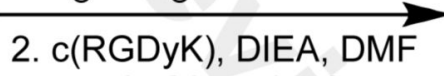

3. TFA/TIS/EDT/ $\mathrm{H}_{2} \mathrm{O}$

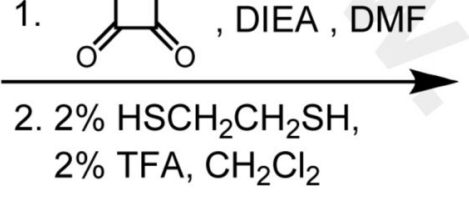

d. Resin 1

1. Glycine, HATU, DIEA, DMF

2. Biotin, HATU, DIEA, DMF 3. TFA/TIS/EDT/ $\mathrm{H}_{2} \mathrm{O}$

e. Resin 1

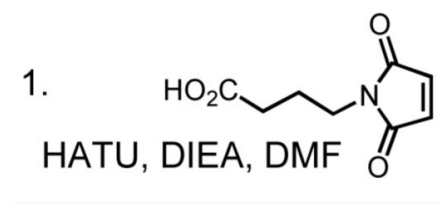

2. $5 \%$ TFA, $1 \% \mathrm{I}_{2}, \mathrm{CH}_{2} \mathrm{Cl}_{2}$<smiles>[R]NC(=O)CNC(=O)[C@@H]1CCCN1C(=O)[C@H](CO)NC(=O)[C@H](CC(=O)O)NC(=O)CNC(=O)[C@H](CCCNC(=N)N)NC(=O)CN</smiles><smiles>[R]Nc1c(NCCCC[C@H]2NC(=O)[C@H](Cc3ccc(O)cc3)NC(=O)[C@H](CC(=O)O)NC(=O)CNC(=O)[C@H]2CCCNC(=N)N)c(=O)c1=O</smiles>

$$
\mathrm{SH} \times \mathrm{\gamma}_{9} \mathrm{O} \times \mathrm{O} \widehat{X}_{3} \xi=\mathrm{r}
$$

Scheme 2.

Applications of resin 1 in AT synthesis. Abbreviations used: TFA-trifluoroacetic acid; TIS — triisopropylsilane; DIEA — N,N-diisopropylethylamine; HATU-2-(1H-7-

azabenzotriazol-1-yl)-1,1,3,3-tetramethyl uronium hexafluorophosphate. 


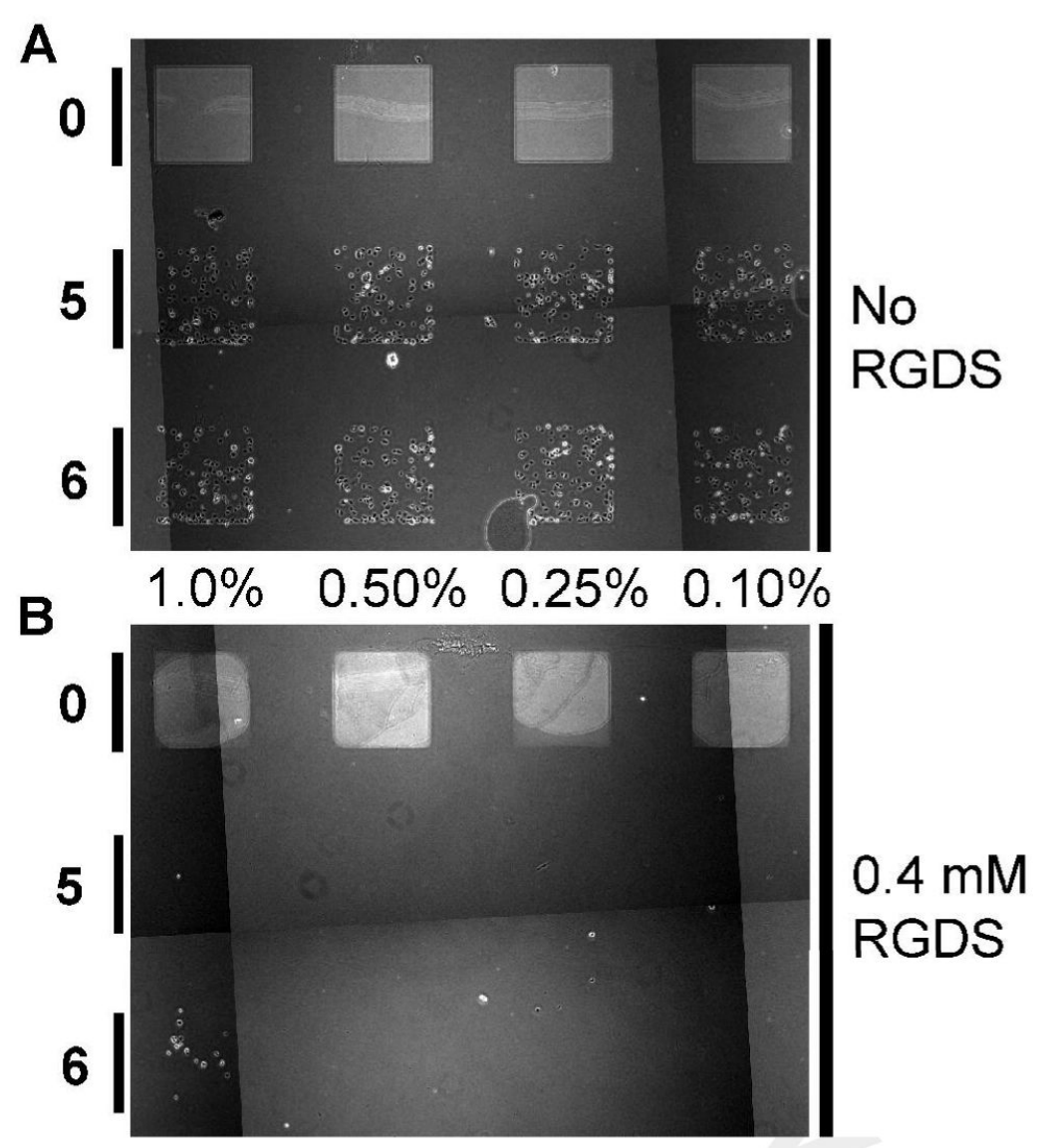

Figure 2.

WM-115 cells adhere specifically to SAMs presenting a linear or cyclic RGD peptide. We fabricated arrays of SAMs that present varying surface densities of GRGDSPG-AT (5) or c (RDGyK)-AT (6). The percentages indicated refer to that of peptide-AT in the mixture with glucamine-AT. ${ }^{4,2}$ The top row of the array elements (labeled $\mathbf{0}$ ) was etched with KCN to yield bare glass surface (to ease comparison and alignment). Arrays were incubated with WM-115 cells for 1 hour in serum-free media containing $0 \mathrm{mM}$ (A) or $0.4 \mathrm{mM}$ GRDS peptide (B). A: Cells adhere only to the surfaces presenting specific binding epitope; they do not adhere to the perfluoro-SAM background or bare glass surface. B: Soluble competitor obliterates cell binding confirming the specificity of the interaction between the SAM and the cells. Cells were fixed, mounted with fluoromount media, and imaged using automated inverted phase-contrast microscope with $4 \mathrm{x}$ objective. The image represents a stitch-mosaic of smaller images. Array element size $-0.8 \times 0.8 \mathrm{~mm}$. 